\title{
Antimicrobial Prophylaxis in Instrumented Spinal Fusion Surgery: A Comparative Analysis of 24-Hour and 72-Hour Dosages
}

\author{
Chandrasekaran Marimuthu ${ }^{1}$, Vineet Thomas Abraham ${ }^{1}$, \\ Mirunalini Ravichandran ${ }^{2}$, Rajamani Achimuthu ${ }^{1}$ \\ ${ }^{1}$ Department of Orthopaedics, Mahatma Gandhi Medical College and Research Institute, Pondicherry, India \\ ${ }^{2}$ Department of Pharmacology, Mahatma Gandhi Medical College and Research Institute, Pondicherry, India
}

\section{Study Design: Prospective study.}

Purpose: To compare the efficacy of 24-hour and 72-hour antibiotic prophylaxis in preventing surgical site infections (SSIs).

Overview of Literature: Antimicrobial prophylaxis in surgical practice has become a universally accepted protocol for minimizing postoperative complications related to infections. Although prophylaxis is an accepted practice, a debate exists with regard to the antibiotic type and its administration duration for various surgical procedures.

Methods: Our institute is a tertiary care hospital with more than 100 spinal surgeries per year for various spine disorders in the department of orthopedics. We conducted this prospective study in our department from June 2012 to January 2015. A total of 326 patients were enrolled in this study, with 156 patients in the 72-hour antibiotic prophylaxis group (group A) and 170 patients in the 24-hour group (group B). Cefazolin was the antibiotic used in both groups. Two surgeons were involved in conducting all the spinal procedures. Our study compared SSIs among patients undergoing instrumented spinal fusion.

Results: The overall rate of SSIs was 1.8\% with no statistical difference between the two groups.

Conclusions: The 24-hour antimicrobial prophylaxis is as effective as the 72-hour dosage in instrumented spinal fusion surgery.

Keywords: Infection surgical site; Fusion spinal; Prophylaxis antibiotic

\section{Introduction}

Antimicrobial prophylaxis in surgical practice has become a universally accepted protocol for minimizing postoperative complications related to infections [1-3]. Surgical site infections (SSIs) are common complications in hospitals. They account for approximately one-fifth of hospitalacquired infections, and rank as the third most common complication in hospitals $[3,4]$. Although prophylaxis is an accepted practice, a debate exists with regard to the antibiotic type to be used and its administration duration for various surgical procedures. Appropriate antibiotic usage will help in reducing the emergence of drug resistance and will also avoid complications such as Clostridium difficile infection arising due to prolonged antibiotic use [5]. The purpose of this study was to compare the efficacy of 24-hour and 72-hour antibiotic prophylaxis in preventing SSIs.

\footnotetext{
Received Apr 3, 2016; Revised May 10, 2016; Accepted Jun 4, 2016

Corresponding author: Chandrasekaran Marimuthu

Spine Unit, Department of orthopaedics, Mahatma Gandhi Medical College and Research Institute,

Pillayarkuppam, Pondicherry, India 607402

Tel:+91-944-3398534, Fax: +91-413-2615457, E-mail: chandruortho@yahoo.com
} 


\section{Materials and Methods}

This was a prospective, randomized, comparative study involving patients undergoing spinal fusion surgery. The study was approved by the institutional research council at our university. All patients above the age of 18 years were included in this study. Patients with suspected spondylodiscitis (pyogenic or tubercular), spinal fusion without stabilization, revision spinal surgery and those who had already received antibiotics for other infections in the past three weeks were excluded from the study. Patients undergoing instrumented spinal fusion were divided into two groups: group A, receiving 72-hour antibiotic prophylaxis, and group B, receiving 24 -hour antibiotic prophylaxis. The prophylactic dosage was initiated at least 30 min before the surgical incision and was continued at 8-hour intervals for 72 or 24 hour, in group A and group $\mathrm{B}$, respectively. Cefazolin injections were used in both groups. If the surgical procedure exceeded 3 hour or if the patient required more than $1,000 \mathrm{~mL}$ of blood transfusion, an additional perioperative dose of $1 \mathrm{~g}$ Cefazolin was administered to the patients. Of the 326 patients, 116 in group A and 128 in group B, who had undergone lumbar and lumbosacral fusion, received an additional intraoperative dose of $1 \mathrm{~g}$ cefazolin as the surgical procedure exceeded 3 hour. No patient in either group received more than $1,000 \mathrm{~mL}$ of blood transfusion. The surgical wound in all patients was closed with a suction drain, which was removed after 48 hour. All patients were catheterized with a Foley catheter, which was removed after 48 hour. Group A had 156 patients and group B had 170 patients. Postoperatively, patients in both groups were closely monitored for signs and symptoms of immediate SSIs, as described by Centers for Disease Control and Prevention, and were followed up for a minimum of one year for late onset SSIs.

\section{Results}

Results were analyzed with regard to age, sex, comorbidities, various fusion levels, and SSIs. The average age was 45.5 and 46.5 years in groups A and B, respectively. No significant differences were noted between the two groups with regard to sex and comorbidities such as diabetes mellitus, smoking and alcoholism. With regard to the spinal fusion levels, there were no statistically significant differences (Table 1, Fig. 1).

Of the 326 patients, 22 were lost to follow-up (group A, 10; group B, 12) after nine months. The remaining 304 patients were followed up for a minimum of one year. With regard to SSIs, six patients who had immediate postoperative infection within two weeks of surgery were diagnosed as per the guidelines of Centers for Disease Control and Prevention. All these infections occurred at the lumbar

Table 1. Patients demographic and surgical profile

\begin{tabular}{|c|c|c|c|}
\hline Demographic data & Group A (n=156) & Group B $(n=170)$ & Statistical $p$-value \\
\hline Age (yr) & 45.5 & 46.5 & $0.8^{\mathrm{a})}$ \\
\hline Sex & & & $0.9^{b)}$ \\
\hline Male & 40 & 42 & \\
\hline Female & 116 & 128 & \\
\hline Diabetes mellitus & 32 & 37 & $0.7^{\mathrm{b})}$ \\
\hline Smoking & 21 & 24 & $0.8^{b)}$ \\
\hline Alcoholism & 10 & 12 & $0.82^{b)}$ \\
\hline \multicolumn{4}{|l|}{ Operation region } \\
\hline Cervical & 22 & 26 & $0.87^{b)}$ \\
\hline Dorsal, dorsolumbar & 18 & 16 & $0.5^{\mathrm{b})}$ \\
\hline Lumbar & 76 & 81 & $0.9^{b)}$ \\
\hline Lumbosacral & 40 & 47 & $0.7^{\mathrm{b})}$ \\
\hline Surgical site infection & 4 & 2 & $0.43^{\mathrm{bl}}$ \\
\hline
\end{tabular}

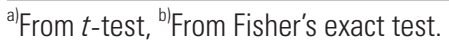


level fusion. These patients were all positive for bacterial growth based on the wound cultures (Fig. 2). Of the six patients, methicillin-resistant Staphylococcus aureus (MRSA) was isolated from three; coagulase-negative Staphylococcus, from two; and "gram-negative" non-fermenting bacilli, from one (Table 2). In all except one, the SSI was classified as a deep infection as evidenced by deep wound gaping and required wound debridement and irrigation along with appropriate antibiotics for a period of six weeks postoperatively. The one patient who had a non-fermenting, gramnegative bacilli infection was diagnosed with a superficial infection and was treated for four weeks with appropriate antibiotics as per the sensitivity pattern of the pathogen. All six patients were followed up for 18 months with no evidence of infection recurrence at the surgical site. The overall SSI rate was $1.8 \%$. SSI incidence was higher in group $\mathrm{A}$, at $2.5 \%$, than in group $\mathrm{B}$, at $1.1 \%$ (Table 3 ).

\section{Discussion}

SSI is a major complication for both the patient and the treating physician irrespective of the surgical procedure as it increases morbidity and mortality associated with

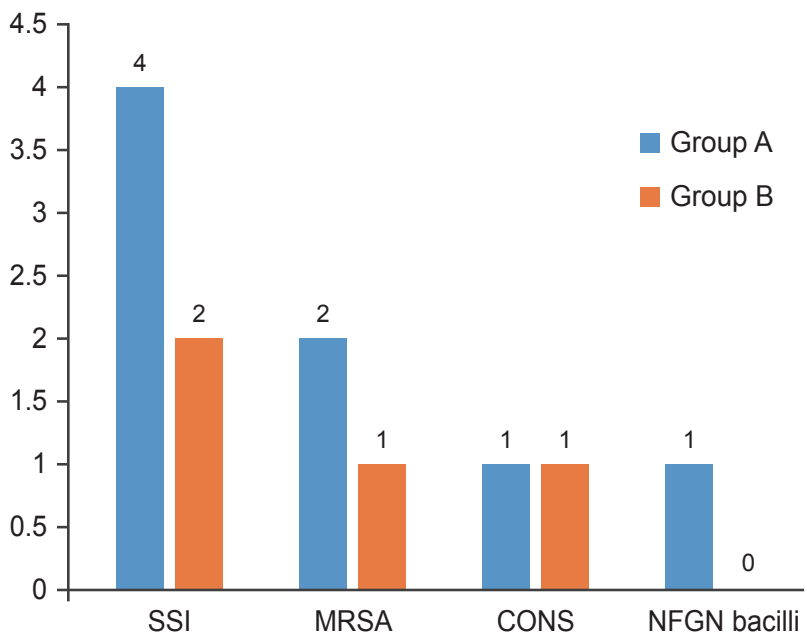

Fig. 2. Pathogenic microorganisms. SSI, surgical site infection; MRSA, methicillin resistant Staphylococcus aureus; CONS, coagulase negative staphylococcus aureus; NFGN bacilli, non-fermenting gram negative bacilli.
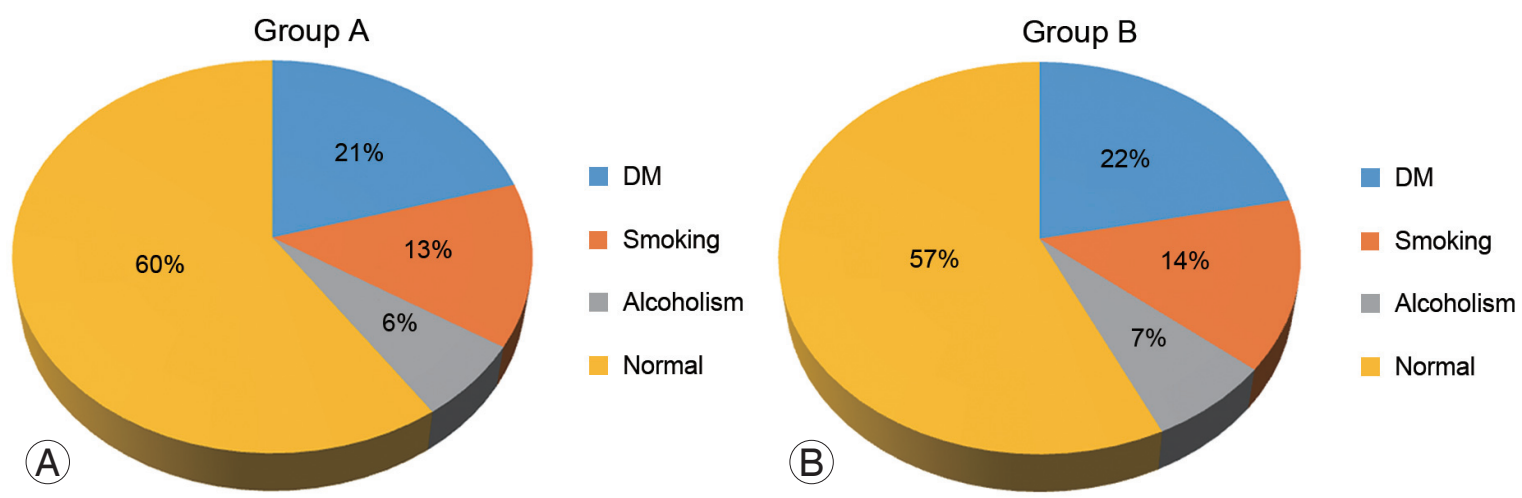

Fig. 1. Demographic profile, group A and group B. (A) Patient demographic profile in group A. (B) Patient demographic profile in group B. DM, diabetes mellitus.

Table 2. Data on SSI patients

\begin{tabular}{|c|c|c|c|c|c|c|c|}
\hline Case & Group & Age/Sex & Diagnosis & Surgery & Comorbidity & Infection type & Organism \\
\hline 1. & A & 55/Female & L4, 5 Lytic listhesis & Instrumented fusion & DM & Deep incisional & MRSA \\
\hline 2. & A & 52/Female & L4, 5 Lytic listhesis & Instrumented fusion & $\mathrm{DM}$ & Deep incisional & MRSA \\
\hline 3. & A & 45/Female & L5, S1 Lytic listhesis & Instrumented fusion & Nil & Deep incisional & CONS \\
\hline 4. & A & 53/Mmale & L4, 5 Degen listhesis & Instrumented fusion & Nil & Superficial incisional & NFGN bacilli \\
\hline 5. & $\mathrm{~B}$ & 58/Female & L4, 5 Degen listhesis & Instrumented fusion & $\mathrm{DM}$ & Deep incisional & MRSA \\
\hline 6. & $\mathrm{~B}$ & 52/female & L5, S1 Lytic listhesis & Instrumented fusion & Nil & Deep incisional & CONS \\
\hline
\end{tabular}

SSI, surgical site infection; DM, diabetes mellitus; MRSA, methicillin resistant Staphylococcus aureus; CONS, coagulase negative staphylococcus aureus; NFGN bacilli, non-fermenting gram negative bacilli. 
Table 3. SSI rate

\begin{tabular}{lccc} 
Group & Infections (SSI) & Uninfected & Total \\
Group A & $4(2.5 \%)$ & 152 & 156 \\
Group B & $2(1.1 \%)$ & 168 & 170 \\
\hline Total & $6(1.8 \%)$ & 320 & 326 \\
\hline
\end{tabular}

SSI, surgical site infection.

the surgery $[3,6,7]$. This is accentuated by the presence of foreign materials such as implants and synthetic grafts at the surgical site. Instrumented spinal fusion is a common, gold standard procedure for various spinal disorders, which involves implants and auto or allo bone grafts/ bone substitutes and carries risks for SSIs similar to any other orthopedic procedure [8-10]. Infection after spinal surgery is a major complication, and every effort should be made by the treating physician and the patient to prevent and/or minimize it. Prophylactic antibiotics play an important role for preventing or minimizing SSIs [9-13]. The incidence of SSIs following adult spinal surgery varies from $0.7 \%$ to nearly $20 \%$, depending on the type of surgical procedure. Most SSIs are caused by Staphylococcus species, in particular S. aureus and S. epidermidis, followed by MRSA. These organisms are either endogenous or exogenous in origin. Most of the time the infection is due to an exogenous contamination [12,14,15]. A suitable antibiotic for an appropriate period is mandatory, but prolonged use of prophylactic antibiotics should be avoided [16-19]. There are many articles emphasizing compliance which show that surgical care improvement projects do minimize or influence SSIs $[19,20]$. A retrospective study conducted by Collins et al. [12], showed that 1,980 instrumented spinal fusions performed over a period of 10 years had an infection rate of $3.7 \%$. S. aureus was the most commonly isolated organism, followed by Propionibacterium. In a recent retrospective analysis by Liu et al. [20], 1,176 patients who underwent open, instrumented lumbar fusion were shown to have a deep infection rate of $2.64 \%$, where most commonly isolated organism was $S$. aureus, followed by MRSA. In a study by Kim et al. [21], the efficacy of 48-hour antimicrobial prophylaxis was compared with that of 72-hour dosage, and it was concluded that for clean spinal surgery, antimicrobial prophylaxis for 48 hours is as efficient as that for 72 hours.

Our study compared the effectiveness of 24-hour and 72-hour antimicrobial prophylaxis, which is unique, as controversies still exist with regard to the duration of antimicrobial prophylaxis in instrumented spinal fusion. In this study, we found no statistical difference between the two groups with regard to SSIs. The overall SSI rate was $1.8 \%$, which is comparable to that reported in many other studies. The 24-hour antimicrobial prophylaxis group had SSI levels compared with the 72-hour group. In developing countries, surgeons still believe that a longer duration of antibiotic administration is required to prevent or minimize SSIs; this includes India, where many surgeons continue to give prophylactic antibiotics up to five days postoperatively. Although the patient volume in our study is small, it is still substantial as we included only instrumented spinal fusion procedures, and managed to convey a strong message that 24 -hour antimicrobial prophylaxis is as effective as the 72-hour dosage.

The limitations of our study are as follows: (1) the total number of patients was relatively small, (2) we did not have absolutely comparable (homogenous) groups, though we tried to match as much as possible, and (3) we did not include sub-analysis to quantify the risk factors for SSIs. This will be the focus in our future study.

\section{Conclusions}

In clean surgeries, like instrumented spinal fusion, 24hour antimicrobial prophylaxis is as effective as the 72hour one.

\section{Conflict of Interest}

No potential conflict of interest relevant to this article was reported.

\section{References}

1. Anderson DJ, Kaye KS, Classen D, et al. Strategies to prevent surgical site infections in acute care hospitals. Infect Control Hosp Epidemiol 2008;29 Suppl 1:S51- 
61.

2. Bowater RJ, Stirling SA, Lilford RJ. Is antibiotic prophylaxis in surgery a generally effective intervention? Testing a generic hypothesis over a set of metaanalyses. Ann Surg 2009;249:551-6.

3. Gomez MI, Acosta-Gnass SI, Mosqueda-Barboza L, Basualdo JA. Reduction in surgical antibiotic prophylaxis expenditure and the rate of surgical site infection by means of a protocol that controls the use of prophylaxis. Infect Control Hosp Epidemiol 2006; 27:1358-65.

4. European Centre for Disease Prevention and Control. Annual Epidemiological Report 2013: reporting on 2011 surveillance data and 2012 epidemic intelligence data. Stockholm: ECDC; 2013.

5. Prospero E, Barbadoro P, Marigliano A, Martini E, D’Errico MM. Perioperative antibiotic prophylaxis: improved compliance and impact on infection rates. Epidemiol Infect 2011;139:1326-31.

6. Akalin HE. Surgical prophylaxis: the evolution of guidelines in an era of cost containment. J Hosp Infect 2002;50 Suppl A:S3-7.

7. Nichols RL. Preventing surgical site infections: a surgeon's perspective. Emerg Infect Dis 2001;7:220-4.

8. Whitehouse JD, Friedman ND, Kirkland KB, Richardson WJ, Sexton DJ. The impact of surgical-site infections following orthopedic surgery at a community hospital and a university hospital: adverse quality of life, excess length of stay, and extra cost. Infect Control Hosp Epidemiol 2002;23:183-9.

9. Ribeiro M, Monteiro FJ, Ferraz MP. Infection of orthopedic implants with emphasis on bacterial adhesion process and techniques used in studying bacterial-material interactions. Biomatter 2012;2:176-94.

10. Barker FG 2nd. Efficacy of prophylactic antibiotic therapy in spinal surgery: a meta-analysis. Neurosurgery 2002;51:391-400.

11. Calderone RR, Garland DE, Capen DA, Oster H. Cost of medical care for postoperative spinal infections. Orthop Clin North Am 1996;27:171-82.

12. Collins I, Wilson-MacDonald J, Chami G, et al. The diagnosis and management of infection following instrumented spinal fusion. Eur Spine J 2008;17:44550.

13. Rechtine GR, Bono PL, Cahill D, Bolesta MJ, Chrin AM. Postoperative wound infection after instrumentation of thoracic and lumbar fractures. J Orthop Trauma 2001;15:566-9.

14. Beiner JM, Grauer J, Kwon BK, Vaccaro AR. Postoperative wound infections of the spine. Neurosurg Focus 2003;15:E14.

15. Cahill PJ, Warnick DE, Lee MJ, et al. Infection after spinal fusion for pediatric spinal deformity: thirty years of experience at a single institution. Spine (Phila Pa 1976) 2010;35:1211-7.

16. Carignan A, Allard C, Pepin J, Cossette B, Nault V, Valiquette L. Risk of Clostridium difficile infection after perioperative antibacterial prophylaxis before and during an outbreak of infection due to a hypervirulent strain. Clin Infect Dis 2008;46:1838-43.

17. Kasliwal MK, Tan LA, Traynelis VC. Infection with spinal instrumentation: Review of pathogenesis, diagnosis, prevention, and management. Surg Neurol Int 2013;4:S392-403.

18. Garey KW, Dao-Tran TK, Jiang ZD, Price MP, Gentry LO, Dupont HL. A clinical risk index for Clostridium difficile infection in hospitalised patients receiving broad-spectrum antibiotics. J Hosp Infect 2008;70: 142-7.

19. Schimmel JJ, Horsting PP, de Kleuver M, Wonders G, van Limbeek J. Risk factors for deep surgical site infections after spinal fusion. Eur Spine J 2010;19:17119.

20. Liu JT, Liao WJ, Chang CS, Chen YH. Management of deep infection after instrumentation on lumbar spinal surgery in a single institution. Biomed Res Int 2015;2015:842010.

21. Kim B, Moon SH, Moon ES, et al. antibiotic microbial prophylaxis for spinal surgery: comparison between 48 and 72-hour AMP protocols. Asian Spine J 2010;4:71-6. 